Folia Cardiologica 2021

\title{
The importance of early diagnosis and treatment of obstructive sleep apnoea in patients with cardiovascular diseases
}

\author{
Karol Osłowski ${ }^{1}$, Wojciech Kukwa² (D), Daniel Śliż ${ }^{1}$ iD \\ ${ }^{1} 3^{\text {rd }}$ Department of Internal Medicine and Cardiology, Medical University of Warsaw, Poland \\ ${ }^{2}$ Department of Otorhinolaryngology, Faculty of Medicine and Dentistry, Medical University of Warsaw, \\ Czerniakowski Hospital in Warsaw, Poland
}

\section{Abstract}

Obstructive sleep apnoea (OSA) is one of the most common sleep-related breathing disorders. New epidemiological studies show that the disease is much more prevalent in the general population than previously believed. Individuals with cardiovascular diseases are a special group of patients suffering from OSA as the prevalence of the disorder among them is much greater than in the general population, and it can constitute an isolated risk factor for the development of some cardiovascular diseases. This article will discuss methods for diagnosing OSA, its epidemiology among patients with particular cardiovascular diseases and the prospects for the development of screening examinations.

Key words: OSA, obstructive sleep apnoea, epidemiology, diagnosis, hypertension, heart failure, atrial fibrillation, coronary heart disease

Folia Cardiologica 2021; 16, 2: 91-96

\section{Introduction}

Physiological sleep is of fundamental importance for the development, regeneration and functioning of the nervous system as well as consolidation of memory pathways. It helps maintain proper cleansing of neurons which prevents later degenerations of the nervous system. It also affects the proper functioning of the cardiovascular system, physiological hormonal play, has an impact on the mood and, indirectly, on each cell of the body. Sleep-related disorders are a multidisciplinary problem which draws the attention of an increasing number of researchers. On the one hand, lack of proper sleep may reduce the productivity of an individual and be a cause of accidents due to impaired concentration during the day. On the other hand, disordered architecture of sleep and some of its pathologies are a factor favouring the development of serious diseases, such as hypertension, atherosclerosis, brain stroke and myocardial infarction [1].

Breathing disorders are among the most serious pathologies of sleep. The term encompasses a number of conditions characterized by various levels of ventilation disorders. According to the guidelines of the American Academy of Sleep Medicine (AASM), which were updated in 2017, we can distinguish such disease entities as: obstructive sleep apnea syndrome, central sleep apnea syndrome, Cheyne-Stokes Respiration, and alveolar hypoventilation syndrome [2].

Address for correspondence: Karol Osłowski MD, III Klinika Chorób Wewnętrznych i Kardiologii, Warszawski Uniwersytet Medyczny, ul. Bursztynowa 2, 04-749 Warszawa, Poland, e-mail: k.p.oslowski@gmail.com

This article is available in open access under Creative Common Attribution-Non-Commercial-No Derivatives 4.0 International (CC BY-NC-ND 4.0) license, allowing to download articles and share them with others as long as they credit the authors and the publisher, but without permission to change them in any way or use them commercially. 
Obstructive sleep apnoea (OSA) is one of the most common sleep-related breathing disorders. It is characterized by multiple episodes of complete collapsing or narrowing of the upper airway lumen in the absence of impaired function of the breathing muscles. They result in a significant or complete cessation of airflow in the respiratory tract. Such episodes are usually accompanied by a decrease in arterial blood oxygen saturation proportional to the duration of apnoea and the degree of airway obstruction. In response to hypoxia, the work of breathing muscles intensifies in order to "break through" the increased resistance of the respiratory tract, which usually results in short, unconscious arousals from sleep. OSA can only be diagnosed if apnoea or shallow breathing last for at least 10 seconds and occur 5 times an hour during sleep. The number of episodes (of apnoea and shallow breathing) per hour is referred to as the apnoea-hypopnoea index ( $\mathrm{AHI})$. On the basis of this index, we can distinguish mild (AHI from 5 to $<15$ ), moderate (AHI from 15 to $<30$ ) and severe $(\mathrm{AHI} \geq 30)$ OSA. Currently, shallowing of breathing accompanied by arousals is also included in the AHI. Scales evaluating sleepiness during the day, such as the Epworth Sleepiness Scale (ESS), are used to determine the severity of the disease; however, due to their low sensitivity, their usefulness in clinical practice is decreasing.

Epidemiological data are vague, most likely due to poor availability of diagnostics as well as insufficient awareness of the problem, among both physicians and patients. Based on the conducted epidemiological studies, it is estimated that the incidence of moderate and severe OSA in the general population ranges from $6 \%$ to $17 \%$, while even $38 \%$ of the population may be suffering from a mild form of the disease [3]. On average, the problem concerns $22 \%$ of men and $17 \%$ of women in all age groups [4]. In HypnoLaus a study involving a large population (3,043 participants), conducted by Swiss researchers - the prevalence of moderate and severe sleep-related breathing disorders (AHI $\geq 15$ ) reached $23.4 \%$ among women and $49.7 \%$ among men [5]. Young men suffer from the disorder 2-3 times more often than women, and the prevalence increases with age. Apart from age and the male sex, risk factors for OSA include obesity, neck circumference equal to or exceeding $43 \mathrm{~cm}$ in men and $40 \mathrm{~cm}$ in women, anatomical abnormalities of the facial skeleton and upper respiratory tract. Risk factors whose association with OSA is less proven include smoking and positive family history of sleep-related breathing disorders. Alcohol, hypnotic drugs and myorelaxants may exacerbate sleep-related breathing disorders, but so far it has not been proven that they contribute to the development of OSA. Greater prevalence than in the general population is also observed in some groups of patients, especially those suffering from congestive heart failure [6], hypertension [7], atrial fibrillation [8], type 2 diabetes [9] and obesity, end-stage renal failure [10] as well as chronic lung diseases and patients in such physiological states as pregnancy [11].

Symptoms of OSA are classically divided into nocturnal and diurnal symptoms. Nocturnal symptoms include loud and irregular snoring, presence of apnoea during sleep (observed by, for example, the partner), nightmares, frequent arousals, difficulties in falling asleep, excessive perspiration and nycturia as well as attacks of dyspnoea during sleep. During the day, patients can be excessively drowsy (even after a seemingly good night's sleep), may observe cognitive decline, decreased libido and sexual potency, throat aches, drying of mucosa as well as tendency towards depression. As mentioned above, obstructive sleep apnoea frequently accompanies other diseases, but can also be responsible for the development thereof. It has been confirmed that OSA is a risk factor for the development of cardiovascular diseases, including brain stroke, ischaemic heart disease and hypertension. Chronic, intermittent hypoxia may lead to activation of the adrenergic and leptinergic system [12] and, in consequence, to metabolic disorders and complications, such as diabetes, obesity and dyslipidemia - regardless of initial obesity [13]. Men suffering from OSA are also significantly more likely to experience erectile dysfunction [14]. Due to the impairment of cognitive abilities, obstructive sleep apnoea has a negative impact on psychophysical abilities of drivers. OSA has been identified in Directive of the European Union of 2014 as one of the most important risk factors for car accidents [15].

While diagnosing a patient in whom OSA is suspected, the physician should take into account their thorough history of sleep and sleep disorders and conduct a complete physical examination of the respiratory, circulatory and nervous system. If the patient reports symptoms that may indicate the occurrence of breathing disorders and risk factors are present, they should be referred for a complete diagnostic evaluation. In the case of OSA, the evaluation includes questionnaires and clinical scales subjectively assessing nocturnal and diurnal symptoms as well as an examination during which sleep-related breathing disorders are recorded. Without an examination consisting in recording sleep-related breathing disorders, clinical evaluation scales are not sufficient to diagnose OSA. For years, polysomnography (PSG) has been the gold standard in diagnostic evaluation. The examination must be conducted in clinical conditions and be supervised by medical staff. With the advancement of technology in the market, portable devices (PM, portable monitoring; HSAT, home sleep apnoea test) for monitoring sleep-related breathing disorders began to appear on the market. Thanks to their simpler design and much better availability, they have become useful tools in diagnostic evaluation of OSA, and in some cases they are sufficient for making the diagnosis. AASM recommends that PSG or an examination with the use of an adequate portable device be conducted in patients without severe 
complications, in whom moderate or severe OSA is suspected on the basis of their symptoms and history [16]. PSG, on the other hand, is the preferred method in patients with severe heart and lung diseases, neuromuscular diseases, insomnia and patients chronically taking opioids. Currently, portable devices are also not recommended for screening examinations or for examinations conducted in patients without symptoms of OSA. A recently published meta-analysis of data [17] calls into question the sensitivity and clinical usefulness of all available methods of screening for OSA in asymptomatic patients. As of the date of writing this article, American Associations do not recommend conducting screening tests in patients without symptoms.

Examinations used in diagnostic evaluation of breathing disorders include:

- classic PSG, supervised throughout the entire night and performed at a sleep lab;

- unsupervised PSG with all channels necessary for the assessment of sleep structure and breathing;

- examinations conducted with the use of instruments monitoring at least 3 physiological variables - respiratory effort, airflow through the upper respiratory tract and blood oxygenation (including the above-mentioned HSAT);

- other examinations during which no more than two variables are recorded.

Of the above examinations, only the classic, supervised PSG cannot be performed on an outpatient basis. The other ones can be also conducted at the patient's home (PM, HSAT). The PSG examination is conducted at a dedicated lab, where the patient stays overnight and is continuously supervised by the medical staff. PSG involves continuous, simultaneous observation and recording of various physiological processes occurring during sleep. It includes electroencephalography (EEG), electrooculography (EOG), electromyography (EMG), electrocardiography (ECG), respiratory flow tests, respiratory effort and blood oxygen saturation measurement. The examination currently constitutes a gold standard for diagnosing OSA. It can also be used to appropriately select treatment parameters for continuous positive airway pressure (CPAP) therapy as well as for post-therapy follow-up.

Portable devices (PM, HSAT) monitoring one to several parameters are also useful in OSA diagnostics. These devices usually differ in terms of their complexity and the amount of measured data, but they are united by common features that distinguish them from classic PSG, namely lower price as well as greater availability and convenience for the patient (no need to spend a night at a medical facility). They may constitute an alternative to PSG in the diagnosis of patients without severe complications, with medical history and symptoms indicating OSA. Diagnosis with the use of HSAT is also suggested for patients requiring urgent diagnosis and prompt introduction of treatment when PSG is not available. According to the guidelines of AASM, portable devices which do not require the supervision of medical staff are sufficient to make diagnosis in patients in whom the probability of moderate and severe OSA is already high before the examination. A comprehensive evaluation of sleep, however, should still be conducted by a specialist. In addition, patients with severe comorbidities cannot be diagnosed with the use of the HSAT method [18].

In 2020, due to the widespread prevalence of OSA in the general population as well as the presumed large underestimation of the problem, six major American associations dealing with sleep-related breathing disorders developed a document that attempted to standardize the nomenclature and juxtapose HSAT devices with the classic PSG as well as their place in contemporary diagnostic evaluation of sleep-related breathing disorders and clinical trials [19]. According to experts, HSAT devices can be useful in diagnosing OSA. In clinical trials evaluating such devices, the profile of the studied patients should be as close as possible to the target group of patients that are likely to benefit from such diagnostics. Panel members also evaluated what parameters should be measured by HSAT. They undoubtedly include saturation and nasal airflow or nasal cavity pressure, heart rate and respiratory effort measurement. It was noticed, however, that the exact number and quality of measurement channels should depend on the clinical issue being examined. Each examination technology, if properly validated with PSG, may prove to be independently effective in the diagnosis of specific breathing disorders during sleep.

\section{OSA and cardiovascular diseases}

Data from large epidemiological studies suggest that obstructive sleep apnoea constitutes and independently significant factor in the development and progress of such cardiovascular diseases as hypertension, heart failure, coronary artery disease as well as various arrhythmias, including atrial fibrillation. Severe OSA increases the risk of both fatal and non-fatal cardiovascular incidents, especially in men. The risk is significantly reduced by CPAP therapy [20].

OSA simultaneously damages the cardiovascular system through several mechanisms - mechanical, chemical and neurohumoral and as a result of an inflammatory response. Decreased intrathoracic pressure, hypoxia and arousal are the direct results of an increased respiratory drive in obstructed airways. "Negative" intrathoracic pressure increases left atrial afterload, causes stretching of atrial walls and increases myocardial oxygen demand which can impair ejection fraction. It is believed that higher incidence of atrial fibrillation in OSA patients is caused by the effect on the mechanoreceptors of atrial walls as well as stretching of the walls. Reflex increase in the sympathetic nervous system tension causes an increase in myocardial 
oxygen demand during impaired gas exchange and hypoxemia, which may cause symptoms of myocardial ischaemia. OSA is also associated with increased oxidative stress and initiation of vascular endothelium-related inflammatory cascade [21]

Observational studies using both PSG and portable devices showed that OSA is more prevalent among heart failure patients with impaired ejection fraction than in the general population (53\% vs. $12 \%$ ) [6]. Cardiac systolic dysfunctions correlate with the degree of OSA, and more commonly occur in a moderate or severe form. It is an interesting observation that in the case of OSA and heart failure patients the diurnal symptoms of OSA are less severe and the ESS scale is not very useful. Data on patients with heart failure and preserved fraction are less available. On the other hand, observational studies proved that CPAP therapy with constant pressure reduced sympathetic activity, blood pressure as well as ESS scores and improved left ventricular systolic function, which may indirectly reduce the risk of readmission to hospital and death [6].

The risk of nocturnal myocardial ischaemia is increased in patients with severe OSA. Breathing disorders and significant desaturation almost double the risk of sudden cardiac death, regardless of other risk factors. OSA patients most often die of sudden cardiac death between midnight and 6 am [21]. Evidence from epidemiological studies indicate that apart from having an impact on the risk factors for ischaemic heart disease (hypertension, atherosclerosis), OSA may also be an independent risk factor for coronary artery disease [22]. The exact mechanism of the phenomenon has not been explained yet, but inflammatory aetiology seems most likely. It is estimated that mild, moderate and severe OSA occurs in, respectively, $69 \%, 43 \%$ and $25 \%$ of patients with acute coronary syndrome [23]. Therefore, it seems legitimate to conduct diagnostics in this group of patients, given the fact that the prognosis is worse if OSA is left untreated [24]. A recent study involving more than 1,000 patients with advanced coronary artery disease eligible for coronary artery bypass grafting (CABG) [25] also showed that moderate and severe OSA was common, occurring in $50.9 \%$ of individuals in this group of patients. The study also proved that in patients with coronary artery disease, OSA was an independent factor contributing to major cardiovascular events, including death.

Another study was published in 2018. It was a meta-analysis within the framework of which major adverse cardiac events (MACE), including death from cardiovascular causes, myocardial infarction, brain stroke, repeated need for coronary revascularization as well as hospitalizations due to heart failure, were evaluated as endpoints [26]. Nine studies, including 2 randomized ones, were analysed. In observational studies, CPAP therapy was associated with a significantly lower risk of MACE, but these data were not confirmed in randomized studies. Treatment of OSA patients with concomitant coronary artery disease can potentially decrease the risk of recurrence of a cardiovascular event, but the role of CPAP in secondary prevention of myocardial infarction is currently being debated due to the lack of expected results in randomized studies [27]. Results of the SAVE study, published in 2016 [28], even suggest that CPAP therapy reduces the intensity of snoring, drowsiness during the day and improves the quality of life and mood, but has no grater impact on the risk of cardiovascular events than standard medical care. These doubts can only be resolved after conducting extensive epidemiological studies concerning the occurrence of OSA in patients with coronary artery disease.

Numerous studies conducted over the years in Europe, the United States and Australia proved that OSA was associated with the development of hypertension. Through the above-mentioned mechanisms, apnoea and hypoxia cause activation of the sympathetic system and spikes in blood pressure, which over time progress to chronic hypertension. In a prospective study involving participants of the Wisconsin Sleep Cohort Study, it was shown after a 4-year-long observation period, which included the impact of age, sex, body build as well as the use of such stimulants as alcohol and cigarettes, that patients with moderate and severe OSA were, respectively, two and three times more likely to develop hypertension than healthy persons [7]. Thus, OSA constitutes an independent factor for the development of hypertension, and the degree of the respiratory disorder correlates with the risk of hypertension. Patients with resistant hypertension, who represent up to a dozen or so percent of patients with diagnosed hypertension, are at a particularly high risk of OSA. Given the number of patients with hypertension in Poland, there may be up to one million individuals with resistant hypertension requiring diagnosis and treatment of OSA.

Arrhythmias are another pathology associated with OSA. Studies using PSG showed that patients with obstructive sleep apnoea experienced nocturnal arrhythmias more often than individuals who do not suffer from this disease. It is estimated that the incidence of atrial fibrillation is approx. $4.8 \%$ higher in OSA patients than in the general population [8]. It seems that diagnosis for respiratory disorders should also be conducted in this group of patients. According to data from randomized studies, the mean HR values and the number of premature ventricular contractions during sleep were lower in patients undergoing CPAP therapy due to OSA. The application of CPAP therapy is also associated with a significant reduction in atrial fibrillation recurrence, even after ablative treatment or electric cardioversion [29]. In a 2015 meta-analysis of data it was proven that the application of CPAP therapy in patients who had undergone an intervention due to atrial fibrillation reduced the risk of $A F$ recurrence by $44 \%$ [30]. OSA is currently believed to 
be a risk factor for AF recurrence and the implementation of OSA diagnostics is recommended in secondary prevention of this arrhythmia [31]. In recent years it has also been observed that various cardiovascular diseases leading to the need for pacemaker implantation are associated with the concomitant presence of undiagnosed OSA. In a multicentre study involving patients with pacemakers implanted due to cardiomyopathy, atrioventricular blocks or sinus node diseases all patients were initially unaware of the fact that they suffered from sleep-related breathing disorders. Based on PSG, obstructive apnoea during sleep was observed in as many as $59 \%$ of patients, and in over $21 \%$ of those patients $\mathrm{AHI}$ exceeded 30 [32]. It shows how common sleep-related breathing disorders are among cardiac patients and how often they can remain undiagnosed by attending physicians.

Currently, patients in Poland are referred for PSG on the basis of symptoms of sleep-related breathing disorders as well as data from medical history. The examination is also recommended in particular disease entities, for example in the case of resistant hypertension and secondary prevention of atrial fibrillation in patients with risk factors. OSA is also ruled out within the framework of the diagnostic and certification process qualifying professional drivers and driving licence applicants who experience symptoms of the disease or have a suggestive history. A doctor specializing in sleep medicine - usually a pulmonary specialist - verifies whether patients are eligible for such an examination. The examination of sleep-related breathing disorders has not been directly included in the guidelines for diagnostics and treatment of the above-described cardiovascular diseases, except for resistant hypertension and secondary prevention of atrial fibrillation. However, taking into account only patients suffering from this disease (estimated at $12-16 \%$ of all hypertensive patients), more than one million potential patients can benefit from PSG. Apart from that, there are also patients with other cardiovascular diseases as well as non-cardiac patients and examinations conducted within the framework of post-treatment follow-up. For economic reasons alone, it is virtually impossible to conduct so many PSG in our health care system. However, portable devices and HSAT systems can help. These solutions are cheaper, more readily available and more convenient for patients. Clinical studies also confirm that despite being slightly less sensitive, HSAT devices are much more cost-effective [33] and can be successfully used for diagnosing OSA. Given the fact that it is not possible to refer a large number of patients for PSG, portable devices seem to be the future of sleep-disordered breathing diagnostics.

Obstructive sleep apnoea is both the cause and a common denominator of many cardiovascular diseases. Current knowledge of this condition indicates that it is underdiagnosed in and the availability of diagnostics and specialist treatment is poor. At this point it seems that the main cause of the above problems in our health care system is the lack of standardization of readily available and inexpensive diagnostic devices that can unburden specialized health care. Devices using modern technologies to detect sleep-related breathing disorders are already available in the market. An example can be Clebre - a wireless acoustic sensor monitoring sleep-related breathing disorders, which continuously analyses recordings of auscultatory phenomena with the use of artificial intelligence algorithms [34]. The device can be used at home and does not have to operated by medical staff. Results of the examination can be sent to the physician immediately after its completion. Nevertheless, further population studies evaluating the effectiveness of such tools as CLEBRE in screening for OSA in specific subpopulations of cardiac patients and others are still needed in order to increase the number of diagnosed OSA cases.

\section{Conflict of interest}

WK - involvement in the Clebre startup, whose R\&D activity is funded by the NCBR's Fast Track grant. The project us co-financed by the European Union from the European Regional Development Fund under the INTERREG Poland - Saxony Cooperation Programme 2014-2020. Project implemented as part of the competition of the NCBR: Fast Track.

\section{References}

1. Lee-Chiong Jr TI. Sleep medicine: essentials and review. Oxford University Press, Oxford 2008.

2. Kapur VK, Auckley D, Chowdhuri S, et al. Clinical practice guideline for diagnostic testing for adult obstructive sleep apnea: an American Academy of Sleep Medicine Clinical Practice Guideline. J Clin Sleep Med. 2017; 13(03): 479-504, doi: 10.5664/jcsm.6506, indexed in Pubmed: 28162150.

3. Senaratna CV, Perret J, Lodge C, et al. Prevalence of obstructive sleep apnea in the general population: A systematic review. Sleep Med Rev. 2017; 34: 70-81, doi: 10.1016/j.smrv.2016.07.002.

4. Franklin KA, Lindberg E. Obstructive sleep apnea is a common disorder in the population - a review on the epidemiology of sleep apnea. J Thorac Dis. 2015; 7(8): 1311-1322, doi: 10.3978/j.issn.20721439.2015.06.11, indexed in Pubmed: 26380759.

5. Heinzer R, Vat S, Marques-Vidal P, et al. Prevalence of sleep-disordered breathing in the general population: the HypnoLaus study. Lancet Respir Med. 2015; 3(4): 310-318, doi: 10.1016/S22132600(15)00043-0, indexed in Pubmed: 25682233.

6. Khattak HK, Hayat F, Pamboukian SV, et al. Obstructive sleep apnea in heart failure: review of prevalence, treatment with continuous positive airway pressure, and prognosis. Tex Heart Inst J. 2018; 45(3): 151-161, doi: 10.14503/THIJ-15-5678, indexed in Pubmed: 30072851.

7. Peppard PE, Young T, Palta M, et al. Prospective study of the association between sleep-disordered breathing and hypertension. N Engl J Med. 2000; 342(19): 1378-1384, doi: 10.1056/ /NEJM200005113421901, indexed in Pubmed: 10805822. 
8. Patel N, Donahue C, Shenoy A, et al. Obstructive sleep apnea and arrhythmia: a systemic review. Int J Cardiol. 2017; 228: 967-970, doi: 10.1016/j.ijcard.2016.11.137, indexed in Pubmed: 27914359.

9. Muraki I, Wada H, Tanigawa T. Sleep apnea and type 2 diabetes. J Diabetes Investig. 2018; 9(5): 991-997, doi: 10.1111/jdi.12823, indexed in Pubmed: 29453905.

10. Voulgaris A, Marrone 0 , Bonsignore MR, et al. Chronic kidney disease in patients with obstructive sleep apnea. A narrative review. Sleep Med Rev. 2019; 47: 74-89, doi: 10.1016/j.smrv.2019.07.001, indexed in Pubmed: 31376590.

11. Truong KK, Guilleminault C. Sleep disordered breathing in pregnant women: maternal and fetal risk, treatment considerations, and future perspectives. Expert Rev Respir Med. 2018; 12(3): 177-189, doi: 10.1080/17476348.2018.1432355, indexed in Pubmed: 29363362.

12. Zakrzewska-Pniewska B, Kwieciński H. Powikłania neurologiczne u chorych na OBPS. Pneumonol Alergol Pol 2007;75. 2007; 75(Suppl 1): 62-64.

13. Patinkin ZW, Feinn R, Santos M. Metabolic consequences of obstructive sleep apnea in adolescents with obesity: a systematic literature review and meta-analysis. Child Obes. 2017; 13(2): 102-110, doi: 10.1089/chi.2016.0248, indexed in Pubmed: 27936906.

14. Kellesarian SV, Malignaggi VR, Feng C, et al. Association between obstructive sleep apnea and erectile dysfunction: a systematic review and meta-analysis. Int J Impot Res. 2018; 30(3): 129-140, doi: 10.1038/s41443-018-0017-7, indexed in Pubmed: 29795528.

15. Siedlecka J, Rybacki M, Pływaczewski R, et al. [The management of obstructive sleep apnea syndrome in drivers - recommendations of the Polish Society Of Occupational Medicine, The Polish Respiratory Society, The Nofer Institute of Occupational Medicine in Lodz and The Polish Sleep Research Society]. Med Pr. 2020; 71(2): 233-243, doi: 10.13075/mp.5893.00927, indexed in Pubmed: 32118870.

16. Rosen IM, Kirsch DB, Carden KA, et al. American Academy of Sleep Medicine Board of Directors, American Academy of Sleep Medicine Board of Directors, American Academy of Sleep Medicine Board of Directors. Clinical use of a home sleep apnea test: an American Academy of Sleep Medicine Position Statement. J Clin Sleep Med. 2017; 13(10): 1205-1207, doi: 10.5664/jcsm.6774, indexed in Pubmed: 28942762.

17. Jonas DE, Amick HR, Feltner C, et al. Screening for obstructive sleep apnea in adults: evidence report and systematic review for the US Preventive Services Task Force. JAMA. 2017; 317(4): 415-433, doi: 10.1001/jama.2016.19635, indexed in Pubmed: 28118460.

18. Clinical guidelines for the use of unattended portable monitors in the diagnosis of obstructive sleep apnea in adult patients. J Clin Sleep Med. 2007; 3(7): 737-747, doi: 10.5664/jcsm.27032.

19. Mann EA, Nandkumar S, Addy N, et al. Study design considerations for sleep-disordered breathing devices. J Clin Sleep Med. 2020; 16(3): 441-449, doi: 10.5664/jcsm.8226, indexed in Pubmed: 31992406.

20. Marin JM, Carrizo SJ, Vicente E, et al. Long-term cardiovascular outcomes in men with obstructive sleep apnoea-hypopnoea with or without treatment with continuous positive airway pressure: an observational study. Lancet. 2005; 365(9464): 1046-1053, doi: 10.1016/S01406736(05)71141-7, indexed in Pubmed: 15781100.

21. Javaheri S, Barbe F, Campos-Rodriguez F, et al. Sleep apnea: types, mechanisms, and clinical cardiovascular consequences. J Am Coll
Cardiol. 2017; 69(7): 841-858, doi: 10.1016/j.jacc.2016.11.069, indexed in Pubmed: 28209226.

22. Wolk R, Kara T, Somers VK. Sleep-disordered breathing and cardiovascular disease. Circulation. 2003; 108(1): 9-12, doi: 10.1161/01. CIR.0000072346.56728.E4, indexed in Pubmed: 12847053.

23. Huang Z, Zheng Z, Luo Y, et al. Prevalence of sleep-disordered breathing in acute coronary syndrome: a systemic review and meta-analysis. Sleep Breath. 2017; 21(1): 217-226, doi: 10.1007/s11325-0161398-9, indexed in Pubmed: 27549104.

24. Jia S, Zhou YJ, Yu Yi, et al. Obstructive sleep apnea is associated with severity and long-term prognosis of acute coronary syndrome. J Geriatr Cardiol. 2018; 15(2): 146-152, doi: 10.11909/j.issn.16715411.2018.02.005, indexed in Pubmed: 29662508.

25. Koo CY, Aung AT, Chen Z, et al. Sleep apnoea and cardiovascular outcomes after coronary artery bypass grafting. Heart. 2020; 106(19): 1495-1502, doi: 10.1136/heartjnl-2019-316118, indexed in Pubmed: 32423904.

26. Wang $X$, Zhang $Y$, Dong $Z$, et al. Effect of continuous positive airway pressure on long-term cardiovascular outcomes in patients with coronary artery disease and obstructive sleep apnea: a systematic review and meta-analysis. Respir Res. 2018; 19(1): 61, doi: 10.1186/ /s12931-018-0761-8, indexed in Pubmed: 29636058.

27. Randerath W, Bonsignore MR, Herkenrath S. Obstructive sleep apnoea in acute coronary syndrome. Eur Respir Rev. 2019; 28(153): 180114, doi: 10.1183/16000617.0114-2018., indexed in Pubmed: 31366458.

28. McEvoy RD, Antic NA, Heeley E, et al. SAVE Investigators and Coordinators. CPAP for prevention of cardiovascular events in obstructive sleep apnea. N Engl J Med. 2016; 375(10): 919-931, doi: 10.1056/ /NEJMoa1606599, indexed in Pubmed: 27571048.

29. Fein AS, Shvilkin A, Shah D, et al. Treatment of obstructive sleep apnea reduces the risk of atrial fibrillation recurrence after catheter ablation. J Am Coll Cardiol. 2013; 62(4): 300-305, doi: 10.1016/ /j.jacc.2013.03.052, indexed in Pubmed: 23623910.

30. Qureshi WT, Nasir UB, Alqalyoobi S, et al. Meta-analysis of continuous positive airway pressure as a therapy of atrial fibrillation in obstructive sleep apnea. Am J Cardiol. 2015; 116(11): 1767-1773, doi: 10.1016/j.amjcard.2015.08.046, indexed in Pubmed: 26482182.

31. Kirchhof P, Benussi S, Kotecha D, et al. ESC Scientific Document Group. 2016 ESC Guidelines for the management of atrial fibrillation developed in collaboration with EACTS. Eur Heart J. 2016; 37(38): 2893-2962, doi: 10.1093/eurheartj/ehw210, indexed in Pubmed: 27567408.

32. Garrigue S, Pépin JL, Defaye P, et al. High prevalence of sleep apnea syndrome in patients with long-term pacing: the European Multicenter Polysomnographic Study. Circulation. 2007; 115(13): 1703-1709, doi: 10.1161/CIRCULATIONAHA.106.659706, indexed in Pubmed: 17353437.

33. Corral J, Mogollon MV, Sánchez-Quiroga MÁ, et al. Spanish Sleep Network, Spanish Sleep Network. Conventional polysomnography is not necessary for the management of most patients with suspected obstructive sleep apnea. Noninferiority, randomized controlled trial. Am J Respir Crit Care Med. 2017; 196(9): 1181-1190, doi: 10.1164/ /rccm.201612-24970C, indexed in Pubmed: 28636405.

34. Mlynczak M, Migacz E, Migacz M, et al. Detecting breathing and snoring episodes using a wireless tracheal sensor - a feasibility study. IEEE J Biomed Health Inform. 2017; 21(6): 1504-1510, doi: 10.1109/ /JBHI.2016.2632976, indexed in Pubmed: 27913363. 